\title{
ON THE ELECTRON MOBILITY AND THE DONOR CENTRES IN REDUCED AND LITHIUM-DOPED RUTILE $\left(\mathrm{TiO}_{2}\right)$
}

\author{
by G. A. ACKET and J. VOLGER \\ Fysisch Laboratorium der Rijksuniversiteit Utrecht, \\ Utrecht, Nederland
}

\section{Synopsis}

Resistivity, Hall- and Seebeck-data of reduced and lithium-doped rutile both with and without alumina added are reported. The data are interpreted by a one band model. Anisotropy of the relaxation time must be taken explicitly into account. The donors in reduced rutile are probably titanium interstitials, which are multiple donors. The first and second of their ionization potentials are derived from the experimental data. Lithium is a single shallow donor. It is demonstrated that aluminium introduces more than one acceptor level. One of these levels is situated close to the conduction band.

A. Introduction. The electrical properties of $n$-type rutile have been studied on strongly reduced $\left.\left.\left.{ }^{1}\right)^{2}\right)^{5}\right)^{18}$ ), slightly reduced $\left.\left.\left.\left.\left.{ }^{3}\right)^{4}\right)^{7}\right)^{8}\right)^{9}\right)^{18}$ ) and niobium-doped ${ }^{2}$ ) crystals. Although the structure of rutile is rather anisotropic, the anisotropy of the conductivity in reduced rutile is only moderate $\left.\left.{ }^{3}\right)^{4}\right)^{18}$ ), and does not depend strongly on temperature. Above $80^{\circ} \mathrm{K}$ the Hall-mobilities do not depend upon the amount of reduction $\left.{ }^{7}\right)^{8}$ ) and are hardly affected by addition of about 0.01 mole $\%$ alumina $\left.\left.\left(\mathrm{Al}_{2} \mathrm{O}_{3}\right)^{5}\right)^{9}\right)$. The Seebeck-coefficient has been studied on strongly reduced ${ }^{2}{ }^{15}$ ) and slightly reduced samples ${ }^{9}$ ). The Hall-coefficient shows anisotropy $\left.\left.{ }^{5}\right)^{7}\right)^{18}$ ), which has been considered indicative of two-band conduction $\left.{ }^{5}\right)^{18}$ ). Recently measurements of the elastoconductivity have been reported ${ }^{17}$ ). The behaviour of the conductivity at high electric field strengths has been studied in slightly reduced samples both at room and liquid air temperatures $\left.{ }^{9}\right)$. Ohmic behaviour was found up to $300 \mathrm{kV} / \mathrm{cm}$. The diffusion of the centres produced by the reduction takes place along the $c$ axis only ${ }^{6}$ ) ${ }^{23}$ ), similar behaviour is found for lithium ${ }^{10}$ ). The dependence of the Hall coefficient of slightly reduced rutile on temperature is strongly influenced by the presence of compensating impurities ${ }^{9}$ ).

It is the purpose of the present paper to report more fully on the electri- 
cal properties of reduced rutile doped with about 0.02 mole $\%$ alumina $\left(\mathrm{Al}_{2} \mathrm{O}_{3}\right)$ and to present data obtained on lithium-doped crystals, both with and without alumina added. A tentative explanation of the mobility and conclusions regarding the nature of the donor centres will be given.

B. Experiments. Rutile crystal boules, both with and without alumina added, were obtained from the National Lead Company, South Amboy, N.J., U.S.A. Spectrochemical analysis indicated a concentration of alumina of about 0.001 mole $\%$ in the undoped crystals and about 0.02 mole $\%$ in the doped crystal (within an accuracy of several tens of percents). Other important impurities were about $0.1 \mathrm{~mole} \% \mathrm{SiO}_{2}$ (which presumably will not be an electrically active centre in rutile) and about 0.001 mole $\% \mathrm{Fe}_{2} \mathrm{O}_{3}$. The orientation of the crystals, which were grown in the 001 direction, was determined by $\mathrm{X}$-ray diffraction and polarization microscopy. Samples were cut with a diamond saw. Reduction was performed by heating specimens in air of $0.1-1 \mathrm{~mm} \mathrm{Hg}$ pressure at $650-800^{\circ} \mathrm{C}$ for one hour. Lithium was diffused into the material by heating in a melt of $\mathrm{Li}_{2} \mathrm{CO}_{3}$ at $750-800^{\circ} \mathrm{C}$ or sometimes by heating samples coated with $\mathrm{LiOH}^{10}$ ) in air of $0.1-1 \mathrm{~mm}$ $\mathrm{Hg}$ pressure at $450^{\circ} \mathrm{C}$. In the former method the samples and melt were cooled down after the treatment in about one minute but even then the samples perpendicular to the $c$ axis lost a considerable amount of lithium by diffusion. It was also checked that no simultaneous production of oxygen deficiency took place during the introduction of lithium. For this purpose bars of 5-10 mm length parallel to the $c$ axis doped with lithium were heated for half an hour at $230-250^{\circ} \mathrm{C}$ in concentrated sulfuric acid, after which treatment they had lost all of their blue colour and conductivity. Reduced samples of similar dimensions, on the other hand, did not show any change of the colour, except at the very ends of the specimen. This is understandable from the difference in diffusivity of lithium ${ }^{10}$ ) and oxygen deficiency ${ }^{6}{ }^{23}$ ). So it is concluded that no appreciablc deviations from stoichiometry are present in our lithium-doped specimens. Lowering of the lithium concentration in bars parallel to the $c$ axis was effectuated by heating in molten $\mathrm{KOH}$ at $330^{\circ} \mathrm{C}$, which procedure serves at the same time as an etching treatment. Of some samples the lithium concentration was determined by diffusing it out, washing the sample and crucible in distilled water with a few drops of $\mathrm{HCl}$ added and subsequent flame-photometric analysis of the solution by means of an Optica flame photometer.

The resistivity and Hall coefficient of reduced samples doped with alumina are shown in figs. 1 and 2. Seebeck-data are given in fig. 3. Resistivity and Hall coefficient of rutile without alumina doped with lithium are presented in figs. 4 and 5, some data obtained on lithium-doped crystals with alumina added are presented in figs. 1 and 2 (LiAl 1 and LiAl 2). It is seen that the Hall mobility $R_{H_{\perp} c / \rho / / c}$ in lithium-doped rutile without 


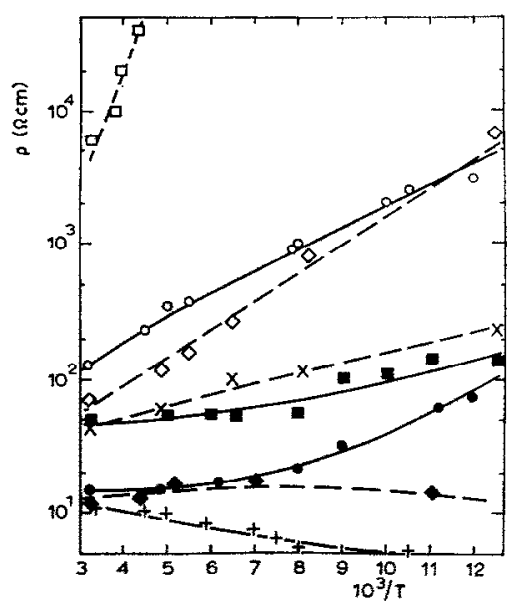

Fig. 1

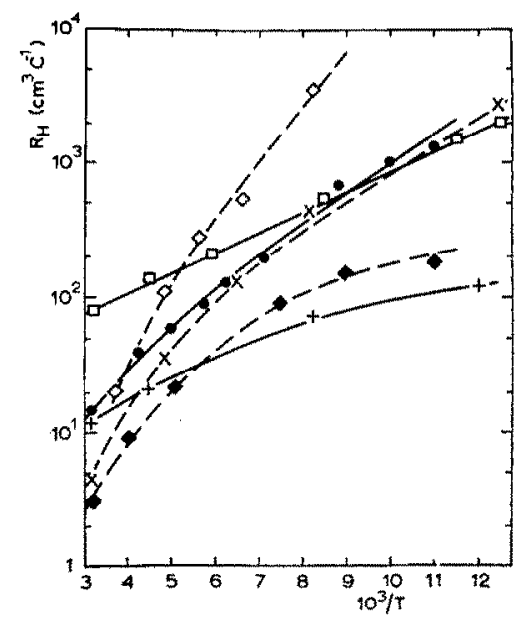

Fig. 12

Fig. 1. Resistivity of reduced and lithium-doped rutile with about 0.02 mole \% alumina added

$(\square \diamond \times \bullet \perp c ; \mathbf{E}+\circ / / c ; \mathrm{RAl}=$ reduced; LiAl = lithium-doped)

- RAl 1, $\times \operatorname{RAl} 2, \diamond \operatorname{RAl} 3, \square \operatorname{RAl} 4,+\operatorname{RAl} 5, \bullet \operatorname{RAl} 6, \circ \operatorname{LiAl} 2, \square L i A l 1$.

Fig. 2. Hall coefficient of reduced and lithium-doped rutile with about 0.02 mole $\%$ alumina added

$(\diamond \times+j \perp c, H / / c ;=+j / / c, H \perp c)$

- RAl 1, $\times \operatorname{RAl} 2, \diamond \operatorname{RAl} 3,+\operatorname{RAl} 5, \bullet \operatorname{RAl} 6, \mathbf{L i A l} 1$.

alumina equals that in reduced rutile ${ }^{5}$ ), while the Hall mobility of lithiumdoped rutile with alumina is also nearly the same. The anisotropy of the resistivity $\rho_{\perp c} / \rho_{/ / c}$ was measured on several reduced samples with alumina. The anisotropy ratio was found to equal $4.5 \pm 0.5$ between $300^{\circ} \mathrm{K}$ and $100^{\circ} \mathrm{K}$, which is not essentially different from the ratios obtained in pure reduced rutile $\left.{ }^{3}\right)^{4}{ }^{7}{ }^{18}$ ). The anisotropy of the Hall coefficient in lithium-

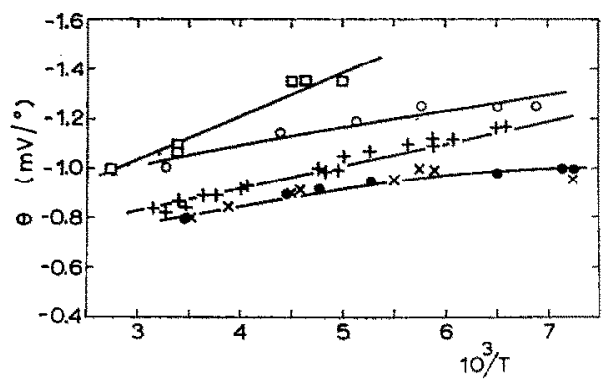

Fig. 3. Sccbcck coefficient of reduced rutile with about 0.02 mole $\%$ alumina added

- RAl 1, $\times \operatorname{RAl} 2, \square \operatorname{RAl} 4,+\operatorname{RAl} 7\left(2 \times 10^{2} \Omega \mathrm{cm} \perp c\right.$ at room temperature $)$, $\circ \mathrm{RAl} 8\left(1 \times 10^{3} \Omega \mathrm{cm} \perp c\right.$ at room temperature $)$. 


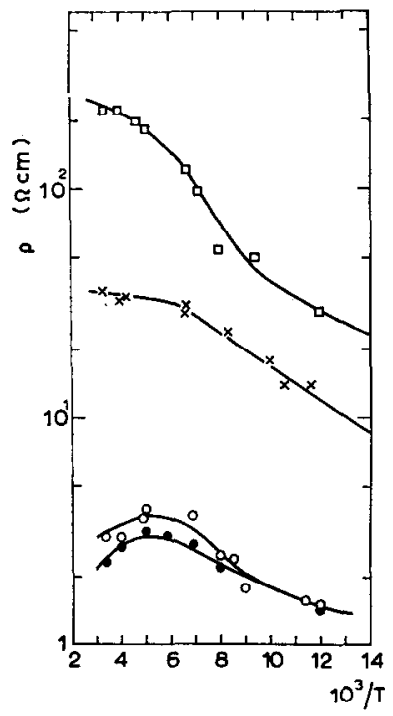

Fig. 4

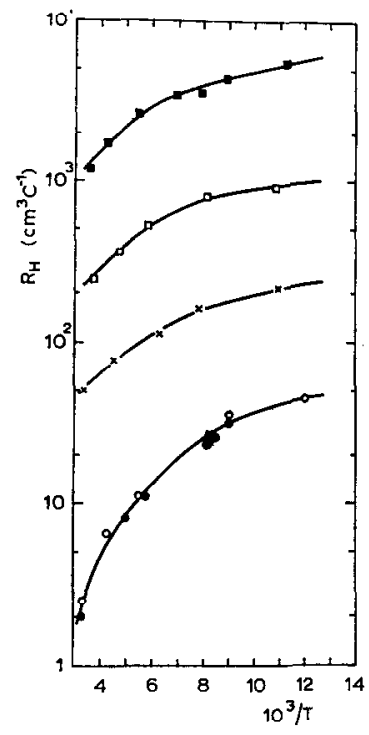

Fig. 5

Fig. 4. Resistivity // $c$ of lithium-doped rutile (without alumina added) - Li 1, O Li 2, x Li 3, $\square \operatorname{Li} 4$.

Fig. 5. Hall coefficient ( $j / / c, H \perp c)$ of lithium-doped rutile (without alumina added) - $\operatorname{Li} 1$, ○ Li 2, x Li 3, 口 Li 4, $\square \operatorname{Li} 5$.

doped $\mathrm{TiO}_{2}$ was studied between $300^{\circ} \mathrm{K}$ and $80^{\circ} \mathrm{K}$ by measuring $R_{H}(j \perp c$; $H / / c)$ on a sample cut perpendicular to the $c$ axis of $33 \Omega \mathrm{cm}$ at room temperature and $R_{H}(j \| c ; H \perp c)$ on a bar cut parallel to the $c$ axis of $8 \Omega \mathrm{cm}$ at room temperature. Because of the anisotropy ratio of the resistivity the lithium-concentrations of these samples should be nearly equal. Indeed the ratio of the Hall coefficients was very close to the Hall coefficient anisotropy ratios of reduccd rutile ${ }^{7}{ }^{18}$ ). (It should be rcmarkcd that this method of determination of the anisotropy ratio was chosen since the very high diffusivity of lithium $/ / c^{10}$ ) makes loss of lithium from thin specimens cut perpendicular to the $c$ axis to occur readily).

From the Hall coefficient and Seebeck data of samples RAl 1 and RAl 2 the density of states effective masses may be derived. It is found that $m^{*}=5-8 m_{e}$ at $300^{\circ} \mathrm{K}$, depending only slightly upon temperature, if the transport contribution to the Seebeck coefficient is assumed to equal $2.5 \mathrm{k} / \mathrm{e}$. (If instead a contribution of $2 \mathrm{k} / \mathrm{e}$ is assumed, masses of 9-13 $m_{e}$ are found). These masses are comparable to those obtained on slightly reduced pure rutile ${ }^{9}$ ), but are lower compared to those obtained by other authors on material without alumina of lower resistivity $\left.{ }^{2}\right){ }^{15}$ ). So it is concluded that the effective masses are not affected by doping with 0.02 mole $\%$ alumina. 
C. Interpretation. Because of the anisotropy of the IIall coetficient it is necessary to investigate what reasonably can be expected about the Hall factors $\left(\mu_{H} / \mu_{D}\right)$. The two-band model by Becker and Hosler $\left.{ }^{18}\right)$ requires that the energy difference between the bottoms of the two conduction bands proposed by these authors be about $0.04 \mathrm{eV}$, which requires that the product of density of states and mobility of the higher band must exceed the same product for the lower band by more than two orders of magnitude (cf. eq. 11 of ref. ${ }^{18}$ ), so that at temperatures higher than $500^{\circ} \mathrm{K}$ the conduction is determined completely by the higher band. Below $40^{\circ} \mathrm{K}$ only the lower band should contribute ${ }^{18}$ ), so that the anisotropy of the mobility of the lower band equals the anisotropy ratio of the resistivity at low temperatures $\left.\left(\rho_{\perp} c / \rho_{/ / c} \cong 2\right)^{18}\right)$. From the magnitude of parameter $C$ of ref. 18 it follows that the anisotropy of the mobility of the higher band should be rather large, which is difficult to reconcile with the anisotropy of the resistivity found at high temperatures $\left.\left.{ }^{7}\right)^{18}\right)\left(600-700^{\circ} \mathrm{K}\right)$ where the conduction is determined by the higher band. This could indicate that the parameter $C$ in Becker and Hosler's paper ${ }^{18}$ ) should not be taken independent of temperature and that the possibility of anisotropy of the relaxation time dependent on temperature should be included. Eagles pointed out ${ }^{11)}$ that for ellipsoids with large longitudinal masses such anisotropy should exist since electrons travelling in the longitudinal directions can only be scattered effectively by phonons having large wave-vectors in these directions. The Seebeck-coefficient is isotropic ${ }^{15}{ }^{23}$ ), which is not generally to be expected for two-band conduction 13 ). Furthermore, measurements performed by $Z$ ijlstra, Leeuwerik and Kleinpenning on generation-recombination noise in reduced rutile doped with alumina indicate that the drift mobility differs from the Hall mobility by at most a factor two between $300^{\circ} \mathrm{K}$ and $77^{\circ} \mathrm{K}^{19}$ ).

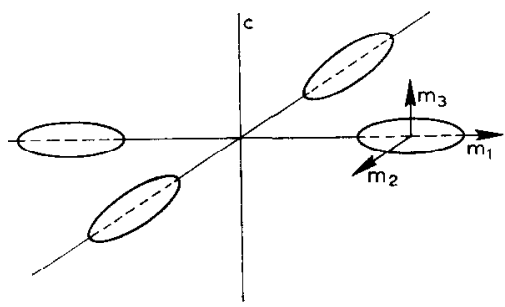

Fig. 6. Structure of the conduction band as proposed for rutile.

For these reasons we have explored an alternative model, viz. a oneband multivalley model as shown in fig. 6 , assuming the longitudinal mass $m_{1}^{*} \approx 50 m_{e}$ and the transverse masses $m_{2}^{*}$ and $m_{3}^{*}$ of the order of the free electron mass. This structure seems reasonable in view of the analogy with $\left.\mathrm{SrTiO}_{3}{ }^{12}\right)$. For simplicity we assume the ellipsoids to point into the $a$ axis 
directions of $k$ space, but other directions of similar symmetry could equally well be possible without any important consequences. At not too low temperature the longitudinal relaxation time $\tau_{1}$ is dominated by phonons of large $k$ vector and so appreciable energy, since only these phonons are capable to produce appreciable change of electron impulse. On the other hand, the transverse relaxation times $\tau_{2}$ and $\tau_{3}$ are dominated by phonons of much smaller wave vector (predominantly low-energy acoustic phonons at not too high temperature), since the impulse is easily randomized within the $m_{2}-m_{3}$ plane. At very high temperatures, $\left(\sim 1000^{\circ} \mathrm{K}\right)$ the scattering will probably be nearly isotropic, but on cooling the longitudinal relaxation time $\tau_{1}$ is expected to increase at a much faster rate than the transverse relaxation times and probably the former will reach its limit imposed by impurity scattering at a higher temperature compared to the transverse relaxation times.

The Hall tensor for the band model of fig. 6 is readily derived using the theory by Herring and $\operatorname{Vog} \mathrm{t}^{14}$ ) which takes anisotropic scattering into account. The elements of the Hall tensor, $R_{H \perp c}$ and $R_{H / / c}$ are given by

$$
R_{H / / c}=\frac{\sigma_{123}}{\sigma_{11 \sigma_{22}}} \quad R_{H / / a}=\frac{\sigma_{231}}{\sigma_{11} \sigma_{33}} .
$$

Here the indices 1 and 2 denote the $a$ directions in $k$ space and 3 stands for the $c$ direction,

$$
\begin{aligned}
\sigma_{x y z}=\sum_{(r)} e n^{(r)} \begin{array}{l}
\left\langle\tau_{x} \tau_{y}\right\rangle \\
m_{x}^{*} m_{y}^{-} \\
(H \text { pointing into the } z \text { direction })
\end{array} \\
\sigma_{x x}=\sum_{(r)} e n^{(r)} \frac{\left\langle\tau_{x}\right\rangle}{m_{x}^{*}}
\end{aligned}
$$

the index $r$ running over the ellipsoids. It then follows

$$
\begin{gathered}
R_{H / / c}=\frac{1}{n e} \frac{4 a K}{(1+K)^{2}} ; \quad R_{H_{\perp} c}=\frac{a}{n e}\left(\frac{\alpha}{a} \frac{K}{K+1}+\frac{1}{K+1}\right) \\
a=\frac{\left\langle\tau_{1} \tau_{2} / m_{1}^{*} m_{2}^{*}\right\rangle}{\left\langle\tau_{1} / m_{1}^{*}\right\rangle\left\langle\tau_{2}\right\rangle / m_{2}^{*}} \cong \frac{\left\langle\tau_{1} \tau_{3} / m_{1}^{*} m_{3}^{*}\right\rangle}{\left\langle\tau_{1} / m_{1}^{*}\right\rangle\left\langle\tau_{3}\right\rangle / m_{3}^{*}} \\
\alpha=\frac{\left\langle\tau_{2} \tau_{3}\right\rangle}{\left\langle\tau_{2}\right\rangle\left\langle\tau_{3}\right\rangle} \quad K=\frac{\left\langle\tau_{2}\right\rangle / m_{2}^{*}}{\left\langle\tau_{1} / m_{1}^{*}\right\rangle} .
\end{gathered}
$$

The longitudinal mass $m_{1}^{*}$ has been taken inside of the average since in the high mass direction the band has a width of a few hundredths of an electronvolt only, so that nonparabolicities are expected to be important in that 
direction. We take it, however, that $a$ is independent of temperature to a first approximation. The anisotropy of the Hall constants is given by:

$$
\frac{R_{H / / c}}{R_{H_{\perp} c}}=\frac{4 K}{(K+1)\left(1+K \cdot \frac{\alpha}{a}\right)} .
$$

For $a / \alpha=1$ the results equal those derived for a similar band structure assuming isotropic scattering ${ }^{13}$ ). Reasonable agreement between relation (3) and the Hall coefficient anisotropy ratio as found experimentally (fig. 7) is obtained taking $a / \alpha \cong 3$ and $K$ varying with temperature as shown in fig. 7. This dependence of $K$ on temperature is reasonable in view of the

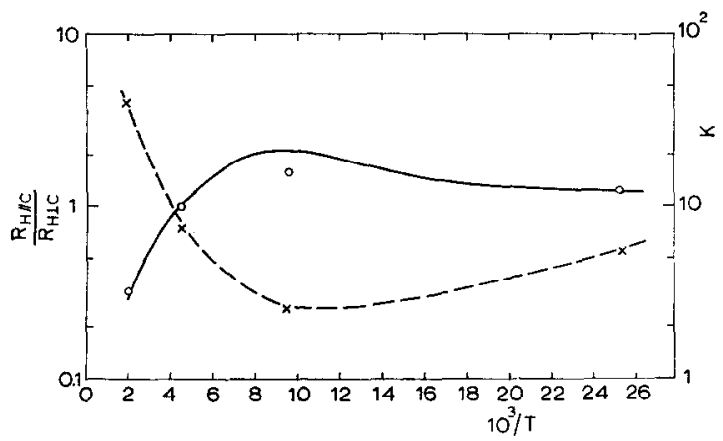

Fig. 7. Anisotropy of the Hall coefficient in $n$-type rutile Full curve: experimental ${ }^{7}{ }^{18}$ ), present work Open circles: theoretical points

Dashed curve: anisotropy parameter $K$ (see eq. (2)) as a function of temperature.

anisotropic scattering by lattice vibrations proposed above. At lower temperatures $\left(\lesssim 40^{\circ} \mathrm{K}\right)$ anisotropic scattering by charged impurities is expected to be important. (It should be remarked that the results of formula (3) do not depend strongly upon the choice of the parameter $a / \alpha$, but $a / \alpha \leq 2$ leads to a maximum of the anisotropy which is too low compared with experiment). If we assume that the relaxation time is isotropic at about $500^{\circ} \mathrm{K}$, the value of the anisotropy parameter at that temperature equals the ratio $m_{1}^{*} / m_{2}^{*}$, which should be about 40 and is expected not to depend strongly upon temperature. According to our model the anisotropy of the relaxation time $\tau_{2} / \tau_{1}$ should be about $\frac{1}{6}$ below $40^{\circ} \mathrm{K}$ which is a reasonable figure compared to the anisotropy for ionized impurity scattering in $n$-type germanium as determined by Laff and Fan from magnetoresistance measurements ${ }^{27}$ ). The Hall factors are easily calculated as a function of temperature from eq. (2) and taking $K$ from fig. 7. Both Hall factors show a maximum at $100^{\circ} \mathrm{K}$, the maximum of $R_{H / / c}$ being the more pronounced. Such behaviour is consistent with the slight maximum in the 
Hall coefficient sometimes found at that temperature $\left.\left.{ }^{2}\right)^{5}\right)$. The Hall factors are compatible with the Seebeck data $\left.\left({ }^{9}\right)^{15}\right)$, present work) and with the data on the drift mobility as obtained from generation-recombination noise ${ }^{19}$ ). From the latter data it appears that it is appropriate to choose $a \cong 3$, so that $\alpha \cong 1$ as expected. The Hall factor corresponding to $R_{H_{\perp} c}$ is then close to unity over the whole temperature region.

We also may derive the anisotropy of the conductivity of the same band model. It is easily found:

$$
\frac{\sigma_{/ / c}}{\sigma_{\perp c}}=\frac{2 K}{K+1} \frac{\frac{\left\langle\tau_{\mathbf{3}}\right\rangle}{m_{3}^{\star}}}{\frac{\left\langle\tau_{2}\right\rangle}{m_{2}^{\star}}} .
$$

Since $\frac{3}{4}<K /(K+1)<1$, we neglect this factor in first approximation, so

$$
\frac{\sigma_{/ / c}}{\sigma_{\perp c}} \cong 2 \frac{\frac{\left\langle\tau_{3}\right\rangle}{m_{3}^{\star}}}{\frac{\left\langle\tau_{2}\right\rangle}{m_{2}^{*}}} .
$$

So our model predicts that the anisotropy of the conductivity be independent of temperature. This is reasonably, although not completely in agreement with experiment $\left.{ }^{3}\right)^{7}$ ). At room temperature it is found then $\left.{ }^{3}\right)^{4}$ )

$$
\frac{\left\langle\tau_{3}\right\rangle m_{2}^{*}}{\left\langle\tau_{2}\right\rangle m_{3}^{*}}=2
$$

which is not far from unity, as expected.

From the temperature-dependence of the Hall mobilities it is then found

$$
\left.\mu_{D / / C} \div\right) T^{-2} \quad T>80^{\circ} \mathrm{K}
$$

This variation is considerably steeper than the dependence like $T^{-1}$ expected for $\tau_{3}$ (acoustic phonon scattering) but this deviation may be understood qualitatively in the following way. At lower temperatures $\boldsymbol{\tau}_{\mathbf{2}}$ and $\tau_{\mathbf{3}}$ are dominated by interaction with phonons having wave vectors within or close to the 2-3-plane, but at higher temperatures scattering into other directions which requires phonons of larger wave vectors will become possible.

Similarly the ohmic behaviour of the conductivity at high field strengths may be understood. Scattering by low-energy acoustic phonons only, leads to deviations from linearity for ${ }^{24}$ )

$$
v_{d}>1.51 c_{l} \text {. }
$$

$v_{d}$ stands for the drift velocity and $c_{l}$ stands for the velocity of the longi- 
tudinal acoustic phonons. From the elastic constants $\left.{ }^{25}\right) c_{l}$ is found to be $0.8-1.2 \times 10^{6} \mathrm{~cm} / \mathrm{s}$, depending upon the direction of propagation. On the other hand, field strengths of $300 \mathrm{kV} / \mathrm{cm}$ at $80^{\circ} \mathrm{K}(/ / c)$ correspond to $v_{d}=$ $=7 \times 10^{6} \mathrm{~cm} / \mathrm{s}$. The linear behaviour of conductivity even at these high field strengths indicates that the process determining the energy relaxation should be rather effective which in our model is well understandable since scattering processes involving phonons of larger wave vector do not necessarily conserve the energy of the electron.

The fact that the mobility near $80^{\circ} \mathrm{K}$ is hardly affected by the presence of 0.02 mole $\%$ alumina $\left(9 \times 10^{18} \mathrm{Al}^{3+}\right.$-ions $\left.\mathrm{cm}^{-3}\right)$ is due to the extremely high dielectric constant $\left(\varepsilon_{c}=170\right.$ and $\left.\varepsilon_{a}=89\right)$ and the large effective mass. Neglecting anisotropy we may estimate the mobility near $80^{\circ} \mathrm{K}$ as determined by ionized impurity scattering by means of the Brooks-Herring equation $\left.{ }^{28}\right)$ :

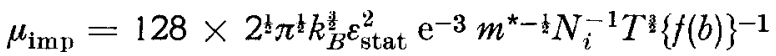

$$
\begin{aligned}
& f(b)=\ln (1+b)-b / 1+b \\
& \left.b=24 \varepsilon_{\text {stat }} k_{B}^{2} \bar{h}^{2} m^{\star} \mathrm{e}^{-2} N_{i}^{\prime}-1 T^{2} \quad N_{i}^{\prime}=n+\left(n+N_{A}\right)\left\{1-\left(n+N_{A}\right) / N_{D}\right)\right\}
\end{aligned}
$$

$k_{B}=$ Boltzmann's constant

$\varepsilon_{\text {stat }}=$ static dielectric constant

$N_{i}=$ concentration of charged centres $\left(\cong 10^{19} \mathrm{~cm}^{-3}\right)$

$\bar{h}=$ Dirac's constant

$N_{A}=$ concentration of acceptor-like centres

$N_{D}=$ concentration of donor centres

$m^{*}=$ effective mass $\left(\cong 10 m_{e}\right)$.

Taking these concentrations to be of the order of $1019 \mathrm{~cm}^{-3}$ it is estimated

$$
\mu_{\mathrm{imp}}\left(80^{\circ} \mathrm{K}\right)=150 \mathrm{~cm}^{2} / \mathrm{V} \mathrm{sec}
$$

which is compatible with our finding that the mobility at $80^{\circ} \mathrm{K}$ is not affected by such a high impurity concentration.

Using the Hall factors derived, information concerning the electron concentration can be obtained from the data on the Hall coefficient (figs. 2 and 4). First, it should be remarked that according to our data lithium is a donor in rutile (since we found lithium-doped material to be $n$-type). From this it is concluded immediately, that lithium is an interstitial impurity, since lithium substituting for titanium should be an acceptor. This conclusion supports the interpretation of the diffusivity of lithium in rutile by Johnson ${ }^{10}$ ). Lithium must be a single donor, its ionization energy can be obtained from the Hall-data of the samples $\mathrm{Li} 1$ and $\mathrm{Li} 2$ since it is reasonable to assume negligible compensation in these samples at least for $T>100^{\circ} \mathrm{K}$. Then

$$
n=\left(N_{D} B_{e}\right)^{\frac{1}{2}} \exp \left(-E_{D} / 2 k T\right)
$$


$N_{D}=$ donor concentration;

$B_{e}$ stands for the density of states of the conduction band

$$
B_{e}=2 \frac{\left(2 \pi m^{*} k T\right)^{\frac{3}{3}}}{h^{3}}
$$

$E_{D}-$ donor ionization energy.

Eq. (6) applies provided $n \ll N_{D}$. Flame-photometric analysis of a sample of similar resistivity yielded

$$
N_{D} \cong 10^{19} \mathrm{Li} \mathrm{cm}^{-3} .
$$

It is then found

$$
E_{D}=0.03 \mathrm{eV} \pm 0.005 \mathrm{eV}
$$

The anisotropy of the diffusivity of the centres produced by reduction ${ }^{6}$ ) ${ }^{23}$ ) indicates that these centres are also interstitials. (The sites available for interstitial species are situated in channels parallel to the $c$ axis ${ }^{20}$ ), which provides a very likely mechanism for the anisotropic diffusion). Moreover, measurements of the oxygen selfdiffusion by $\mathrm{Haul}$ and $\mathrm{Dümbgen}$ performed on samples doped with alumina (150 p.p.m.) ${ }^{16}$ ) are available. From their data it appears that the concentration of the oxygen vacancies responsible for the oxygen diffusion is determined by this impurity content, which indicates that probably cvery moleculc of $\mathrm{Al}_{2} \mathrm{O}_{3}$ introduces one oxygen vacancy into the lattice. From the diffusivity of oxygen the diffusivity of the oxygen vacancies is easily found, since the concentration of the latter is known. Comparison of the oxygen vacancy diffusivity with the diffusivity found experimentally ${ }^{6}{ }^{23}$ ) shows that the latter is higher by orders of magnitude. Furthermore, the diffusivity of the oxygen vacancy is hardly anisotropic ${ }^{16}$ ). So we conclude that the donors in reduced rutile are titanium interstitials, which conclusion is also supported by mechanical loss data ${ }^{21}$ ). In contrast to the lithium interstitials the titanium interstitials are multiple donors, the neutral donor being

$$
\left[\mathrm{Ti}_{\text {int. }}^{n+}, n e\right] \quad n \leqq 4
$$

and the completely ionized donor $\mathrm{Ti}_{\text {int. }}^{4+}$. Here, $\left[\mathrm{Ti}_{\text {int., }}^{n+}, n e\right]$ denotes an interstitial $\mathrm{Ti}^{n+}$-ion together with $n$ electrons in bound states. Only a small energy will be required to remove one electron from this centre. Indeed a shallow donor level has been found ${ }^{5}$ ) at about $0.006 \mathrm{eV}$. We identify this energy with the first ionization potential of our donor-centre i.e. the energy $E_{D}^{(1)}$ required for

$$
\left[\mathrm{T}_{\text {int. }}^{n+}, n e\right] \rightarrow\left[\mathrm{Ti}_{\text {int. }}^{n+},(n-1) e\right]+e
$$

the latter electron being in the conduction band.

Considering the Hall coefficients of samples RAl 1 and RAl 2 it is seen 
that after correction by the appropriate Hall factors, they can be interpreted by the relation

$$
n(:) T^{z} \exp \left(-E_{D}^{(2)} \mid k T\right)
$$

which applies to a set of partially compensated donors in equilibrium with the conduction band. It is known that alumina produces acceptor-like centres in rutile $\left.{ }^{26}\right)$. In slightly reduced specimens the electrons originating from the highest donor levels are taken up by these compensating levels, the former remaining exhausted or only partly filled. In samples RAl 1 and RAl 2 the shallow donor level is clearly exhausted and probably a number of centres will have lost already a second electron. For the energy according to (7) was found

$$
E_{D}^{(2)}=0.025 \pm 0.005 \mathrm{eV} .
$$

We thus attribute this energy to the second ionization potential of the donor centre, i.e. the energy required for the reaction:

$$
\left[\mathrm{Ti}_{\text {int. }}^{n+},(n-1) e\right] \rightarrow\left[\mathrm{Ti}_{\text {int. }}^{n+},(n-2) e\right]+e .
$$

In some slightly compensated samples investigated by Becker and Hosler $\left.{ }^{18}\right)$ comparable activation energies are reported. It is to be noted that

$$
E_{D}^{(2)}=4 E_{D}^{(1)}
$$

This is what is to be expected as long as the potential experienced by the electrons does not deviate too much from the Coulomb potential. This seems reasonable since both levels are rather shallow. If a hydrogenic model is used (which is probably not correct in view of the multi-valley structure assumed for the conduction band) and for the dielectric constant the average of the static dielectric constants $/ / c$ and $\perp c$ are taken it is found

$$
m^{*}=7 m_{e}
$$

which compares very well with the results obtained by us from combined Hall- and Seebeck-data.

Similar analysis of the Hall coefficient of sample RAl 3 indicates the presence of a partly compensated level at $0.05 \mathrm{eV}$. The dependence of the resistivities of samples RAl 3 and LiAl 2 on temperature are very similar, so we conclude that this level is related to high acceptor-like "aluminum"levels. It follows from the work of Yahia ${ }^{26}$ ) that aluminum must be at least a double acceptor in rutile. From the work by $\mathrm{Ha} \mathrm{ul}$ and $\mathrm{D} \mathrm{umbgen}{ }^{16}$ ) it was concluded that together with two $\mathrm{Al}^{3+}$-ions one oxygen-vacancy is introduced into the lattice which then, for sake of neutrality must be devoid of electrons, the resulting centres being $\left[2 \mathrm{Al}^{3+}, \mathrm{O}_{V}\right]$ (the $\mathrm{Al}^{3+}$ substituting for $\left.\mathrm{Ti}^{4+}\right)$. Such centres are able to take up two electrons in the oxygen vacancy, but the energies will be affected by the presence of the $\mathrm{Al}^{3+}$-ions 
in the neighbourhood of the oxygen vacancies, moreover, these $\mathrm{Al}^{3+}{ }^{3+}$-ions, which are negative with respect to the crystal will create a kind of repulsive potential around them. It is of interest to mention that $Z$ ijlstra, Leeuwerik and Kleinpenning ${ }^{19}$ ) found that the capture cross section of the $0.05 \mathrm{eV}$-centre as deduced from generation-recombination noise in reduced aluminium-doped rutile is very small $\left(\sim 10^{-21} \mathrm{~cm}^{2}\right)$ which indeed is indicative of such a repulsive potential.

Part of the phenomena in rutile are thus explained with a simple model. Difficulties remain, however. In our model the anisotropy of the Hall coefficient requires a multi-valley conduction band. Becker and Hosler ${ }^{18}$ ), who used a two-band model for the explanation of this anisotropy, conclude that at least the higher band, which according to their parameters dominates at higher temperatures $\left(500^{\circ} \mathrm{K}-700^{\circ} \mathrm{K}\right)$, should be of the manyvalley type with at least four ellipsoids. Measurements of the elasto-conductive coefficients were recently reported ${ }^{17}$ ) between $77^{\circ} \mathrm{K}$ and $700^{\circ} \mathrm{K}$. The elasto-conductivity coefficients $\left(m_{11}-m_{12}\right) / 2, m_{44}$ and $m_{66}$ were all rather small over the whole temperature range, while in a multi-valley structure with at least four ellipsoids one of these coefficients is expected to be large and proportional to $T^{-1}$. A similar discrepancy is found in $\left.\mathrm{SrTiO}_{3}{ }^{12}\right)^{22}$ ). It was predicted ${ }^{12}$ ), that in $\mathrm{SrTiO}_{3}$ the conduction band is very flat in the 100-directions with the minimum at the zone boundary, which prediction is almost completely based on symmetry. By analogy a multi-valley band should also be probable in rutile ${ }^{12}$ ). The discrepancies in both materials could be due to the following reasons:

1. The deformation potentials could be small.

2. The assumption that by deformation of the lattice the bands (valleys) shift as a whole without change of the effective masses is not sure to hold if the mass in some directions is so large that the bandwidth in these directions is a few hundredths of an electronvolt only.

Although contributions from higher conduction bands cannot be cxcluded at present it has been shown that the experimental data are reasonably explained by a one-band model. The elasto-conductivity coefficients ${ }^{17}$ ) are difficult to interpret in both models. Information concerning the donor centres in reduced and lithium-doped rutile has been obtained: the donors in reduced material are presumably titanium interstitials, which are multiple donors. Lithium is a single shallow donor. The presence of an acceptor level associated with $\mathrm{Al}^{3+}$ situated close to the conduction band was also demonstrated.

Acknowledgements. The authors are indebted to Mr. D. M. Beals of the National Lead Company for delivering the rutile single crystals. We are grateful to Dr. N. W. H. Addink of Philips Laboratories, Eindhoven, the Netherlands, for the spectro-chemical analysis performed under his 
supervision and to Dr. M. H. van Maaren for advice and discussions concerning the experiments with lithium. The rutile crystals were oriented by X-ray diffraction by Mr. J. B. Hulscher of the Laboratorium voor Kristalchemie der Rijksuniversiteit Utrecht.

This work was part of the research programme of the "Stichting voor Fundamenteel Onderzoek der Materie" (FOM) and was made possible by financial support of the "Nederlandse Stichting voor Zuiver Wetenschappelijk Onderzoek" (ZWO).

Received 18-3-66

\section{REFERENCES}

1) Breckenridge, R. G. and Hosler, W. R., Phys. Rev. 91 (1953) 793.

2) Frederikse, H. P. R., J. appl. Phys. Suppl. 32 (1961) 2211.

3) Acket, G. A. and Volger, J., Physica 99 (1963) 225.

4) Bogomolov, V. N. and Shavkunov, P. M., Fizika Tverdogo Tela 5 (1963) 2027.

5) Becker, J. H. and Hosler, W. R., J. Phys. Soc. Japan. Suppl. 18 (1963) 152.

6) Bogomolov, V. N., Fizika Tverdogo Tela 5 (1963) 2011.

7) Bogomolov, V. N. and Zhuse, V. P., Fizika Tverdogo Tela 5 (1963) 3285.

8) Acket, G. A. and Volger, J., Physics Lett. 8 (1964) 244.

9) Acket, G. A. and Volger, J., Physica 30 (1964) 1667.

10) Johnson, O. W., Phys. Rev, 136 (1964) A284.

11) Eagles, D. M., J. Phys. Chem. Solids 25 (1964) 1243.

12) Kahn, A. H. and Leyendecker, A. J., Phys. Rev. 135 (1964) A 1321.

13) Hernandez, W. R. and Kahn, A. H., J. Res. Nat. Bur. Stand. 67A (1964) 293.

14) Herring, C. and Vogt, E., Phys. Rev. 101 (1956) 944.

15) Thurber, W. B. and Mante, A. J. H., Phys. Rev. 139 (1965) A1655.

16) Haul, R. and Dümbgen, G., J. Phys. Chem. Solids 26 (1965) 1.

17) Bir, G. L., Bogomolov, V. N., Krivitskii, E. V. and Suliatitskaya, T. E., Fizika Tverdogo Tela 7 (1965) 2978.

18) Becker, J. H. and Hosler, W. R., Phys. Rev. 137 (1965) A 1872.

19) Zijlstra, R. J. J. and Leeuwerik, F. J., to be published. Zijlstra, R. J. J. and Kleinpenning, Th. G. M., to be published.

20) Hurlen, T., Acta Chimica Scandinavica 13 (1959) 365.

21) Wachtman, J, B. Jr. and Doyle, L. R, Phys. Rev. 135 (1964) A276.

22) Tufte, O. N. and Stelzer, E. L., Bull. Am. Phys. Soc. Ser. II 10 (1965) 305, and Phys. Rev. $141(1966) 675$.

23) Acket, G. A., Thesis, Rijksuniversiteit Utrecht, (1965).

24) Shockley, W., Bell Syst. Techn. J. 30 (1951) 990.

25) Wachtman, J. B. Jr., Tefft, W. F. and Lam, D. G., J. Res. Nat. Bur. Stnds. 66A (1962) 465.

26) Yahia, J., Phys. Rev, 130 (1963) 1711.

27) I. aff, R. A. and Fan, H. Y., Phys. Rev. 112 (1958) 317.

28) Brooks, H., Phys. Rev. 83 (1951) 879. 